\title{
On closure operators related to maximal tricliques in tripartite hypergraphs
}

\author{
Dmitry I. Ignatov
}

\begin{abstract}
Triadic Formal Concept Analysis (3FCA) was introduced by Lehman and Wille almost two decades ago. And many researchers work in Data Mining and Formal Concept Analysis using the notions of closed sets, Galois and closure operators, closure systems, but up-to-date even though that different researchers actively work on mining triadic and n-ary relations, a proper closure operator for enumeration of triconcepts, i.e. maximal triadic cliques of tripartite hypergaphs, was not introduced. In this paper we show that the previously introduced operators for obtaining triconcepts and maximal connected and complete sets (MCCSs) are not always consistent and provide the reader with a definition of valid closure operator and associated set system. Moreover, we study the difficulties of related problems from order-theoretic and combinatorial point view as well as provide the reader with justifications of the complexity classes of these problems.
\end{abstract}

Keywords: Triaidic Formal Concept Analysis, Closure operator, triadic hypergraph, triset, tripartite graphs

\section{Introduction}

Pattern mining is one of the most important Data Mining areas and often relies on fundamental notions from theoretical computer science and algebra like fixpoints, closure operators and lattices (Zaki \& Hsiao, 2005, Boley et al. 2010). Formal Concept Analysis (Ganter \& Wille, 1999) can be considered as an elegant algebraic framework to deal with (frequent) closed sets of objects and

Preprint submitted to Discrete Applied Mathematics

September 16, 2018 
their attributes (formal concepts or maximal bicliques) by means of two closure operators formed by Galois connection over these sets.

Recent studies showed that there are efficient algorithms for building all formal concepts not only in binary object-attribute case but in ternary (TRIAS, (Jäschke et al., 2006)) and $n$-ary cases (DATA-PEeler, (Cerf et al., 2009)).

Several researchers tried to develop a proper closure operator for triadic (Trabelsi et al. 2012) and n-ary cases (Spyropoulou et al., 2014). However the detailed analysis in this paper shows that the concept-forming operator in (Trabelsi et al., 2012) is not always monotone on triset systems. An interesting approach from (Spyropoulou et al., 2014) can be used to enumerate formal triconcepts as the maximal fixpoints of a set system of closed and connected sets (CCS) but suffers from presence of phantom hyperedges because of the lossy $k$-partite graph encoding. In this paper, we show how to define a proper triset system for the concept forming operator from (Trabelsi et al. 2012) that makes it a closure operator, describe the family of closure operators of this type and investigate their properties, and prove that there is no an associated closure operator on the whole triset system for a given tricontext. We also introduce a notion of (maximal) switching generator - a triset resulting in different closed patterns that contain it. In addition we show how to deal with lossy hyperedge encoding and phantom edges to generate triconcepts as maximal connected and complete sets.

The rest of the paper is organised as follows. In Section 2, we recall basic definitions from FCA and its polyadic extensions and reproduce necessary definitions and propositions from (Spyropoulou et al. 2014). In Section 3 , we discuss the studied concept and closed CCS forming operators with a focus on their inconsistency conditions. Section 4 reports our main results. Section 5 discusses related work and and Section 6 concludes the paper. 


\section{Multimodal and multirelational closed patterns}

\subsection{Formal Concept Analysis and its polyadic extensions}

First, we recall some basic notions from Formal Concept Analysis (FCA) (Ganter \& Wille, 1999).

Let $G$ and $M$ be sets, called the set of objects and attributes, respectively, and let $I$ be a relation $I \subseteq G \times M$ : for $g \in G, m \in M, g I m$ holds iff the object $g$ has the attribute $m$. The triple $\mathbb{K}=(G, M, I)$ is called a (formal) context.

A triadic context $\mathbb{K}=(G, M, B, Y)$ consists of sets $G$ (objects), $M$ (attributes), and $B$ (conditions), and ternary relation $Y \subseteq G \times M \times B$ (Lehmann $\&$ Wille, 1995). An incidence $(g, m, b) \in Y$ shows that the object $g$ has the attribute $m$ under the condition $b$.

An $n$-adic context is an $(n+1)$-tuple $\mathbb{K}=\left(K_{1}, K_{2}, \ldots, K_{n}, Y\right)$, where $Y$ is an $n$-ary relation between sets $K_{1}, \ldots, K_{n}$ (Voutsadakis, 2002).

\subsubsection{Concept forming operators and formal concepts}

If $A \subseteq G, B \subseteq M$ are arbitrary subsets of objects and attributes, respectively, then the Galois connection is given by the following derivation operators:

$$
\begin{aligned}
& A^{\prime}=\{m \in M \mid g I m \text { for all } g \in A\}, \\
& B^{\prime}=\{g \in G \mid g I m \text { for all } m \in B\} .
\end{aligned}
$$

If we have several contexts, the derivation operator of a context $(G, M, I)$ is denoted by $(.)^{I}$.

The pair $(A, B)$, where $A \subseteq G, B \subseteq M, A^{\prime}=B$, and $B^{\prime}=A$ is called a (formal) concept (of the context $\mathbb{K}$ ) with extent $A$ and intent $B$ (in this case we have also $A^{\prime \prime}=A$ and $\left.B^{\prime \prime}=B\right)$.

The concepts, ordered by $\left(A_{1}, B_{1}\right) \geq\left(A_{2}, B_{2}\right) \Longleftrightarrow A_{1} \supseteq A_{2}\left(B_{2} \supseteq B_{1}\right)$, form a complete lattice, called the concept lattice $\underline{\mathfrak{B}}(G, M, I)$. 


\subsubsection{Formal concepts in triadic and in $n$-ary contexts}

For convenience, a triadic context is denoted by $\mathbb{K}=\left(X_{1}, X_{2}, X_{3}, Y\right)^{1}$, A triadic context $\mathbb{K}=\left(X_{1}, X_{2}, X_{3}, Y\right)$ gives rise to the following dyadic contexts

$$
\mathbb{K}^{(1)}=\left(X_{1}, X_{2} \times X_{3}, Y^{(1)}\right), \mathbb{K}^{(2)}=\left(X_{2}, X_{1} \times X_{3}, Y^{(2)}\right), \mathbb{K}^{(3)}=\left(X_{3}, X_{1} \times\right.
$$
$\left.X_{2}, Y^{(3)}\right)$,

where $g Y^{(1)}(m, b): \Leftrightarrow m Y^{(2)}(g, b): \Leftrightarrow b Y^{(3)}(g, m): \Leftrightarrow(g, m, b) \in Y$. The derivation operators (primes or concept-forming operators) induced by $\mathbb{K}^{(i)}$ are denoted by $(.)^{(i)}$. For each induced dyadic context we have two kinds of such derivation operators. That is, for $\{i, j, k\}=\{1,2,3\}$ with $j<k$ and for $Z \subseteq X_{i}$ and $W \subseteq X_{j} \times X_{k}$, the $(i)$-derivation operators are defined by:

$$
\begin{gathered}
Z \mapsto Z^{(i)}=\left\{\left(x_{j}, x_{k}\right) \in X_{j} \times X_{k} \mid x_{i}, x_{j}, x_{k} \text { are related by } \mathrm{Y} \text { for all } x_{i} \in Z\right\} \\
W \mapsto W^{(i)}=\left\{x_{i} \in X_{i} \mid x_{i}, x_{j}, x_{k} \text { are related by } \mathrm{Y} \text { for all }\left(x_{j}, x_{k}\right) \in W\right\}
\end{gathered}
$$

Formally, a triadic concept of a triadic context $\mathbb{K}=\left(X_{1}, X_{2}, X_{3}, Y\right)$ is a triple $\left(A_{1}, A_{2}, A_{3}\right)$ of $A_{1} \subseteq X_{1}, A_{2} \subseteq X_{2}, A_{3} \subseteq X_{3}$ such that for every $\{i, j, k\}=$ $\{1,2,3\}$ with $j<k$ we have $\left(A_{j} \times A_{k}\right)^{(i)}=A_{i}$. For a certain triadic concept $\left(A_{1}, A_{2}, A_{3}\right)$, the components $A_{1}, A_{2}$, and $A_{3}$ are called the extent, the intent, and the modus of $\left(A_{1}, A_{2}, A_{3}\right)$. It is important to note that for interpretation of $\mathbb{K}=\left(X_{1}, X_{2}, X_{3}, Y\right)$ as a three-dimensional cross table, according to our definition, under suitable permutations of rows, columns, and layers of the cross table, the triadic concept $\left(A_{1}, A_{2}, A_{3}\right)$ is interpreted as a maximal cuboid full of crosses.

The set of all triadic concepts of $\mathbb{K}=\left(X_{1}, X_{2}, X_{3}, Y\right)$ is denoted by $\mathfrak{T}(\mathbb{K})$. However this set does not form a partial order by extent inclusion since it is possible for the same triconcept extent to have different combinations of intent and modus components (Wille, 1995, Lehmann \& Wille, 1995), similarly, for orderings along the attribute and condition components.

\footnotetext{
${ }^{1}$ Note that in the title we refer to a formal tricontext as a tripartite hypergraph since we deal with three types of vertices connected by triadic hyperedges.
} 
There is a quasiorder $\lesssim_{i}$ for each $i \in\{1,2,3\}$ and its corresponding equivalence relation $\sim_{i}$ is defined by

$$
\begin{gathered}
\left(A_{1}, A_{2}, A_{3}\right) \lesssim_{i}\left(B_{1}, B_{2}, B_{3}\right): \Longleftrightarrow A_{i} \subseteq B_{i} \text { and } \\
\left(A_{1}, A_{2}, A_{3}\right) \sim_{i}\left(B_{1}, B_{2}, B_{3}\right): \Longleftrightarrow A_{i}=B_{i} .
\end{gathered}
$$

These quasiorders satisfy the antiordinal dependencies (Wille, 1995): For $\{i, j, k\}=\{1,2,3\}$ and all triconcepts $\left(A_{1}, A_{2}, A_{3}\right)$ and $\left(B_{1}, B_{2}, B_{3}\right)$ from $\mathfrak{T}(\mathbb{K})$ it holds that $\left(A_{1}, A_{2}, A_{3}\right) \lesssim_{i}\left(B_{1}, B_{2}, B_{3}\right)$ and $\left(A_{1}, A_{2}, A_{3}\right) \lesssim_{j}\left(B_{1}, B_{2}, B_{3}\right)$ imply $\left(A_{1}, A_{2}, A_{3}\right) \gtrsim_{k}\left(B_{1}, B_{2}, B_{3}\right)$.

One may introduce $n$-adic formal concepts without $n$-ary concept forming operators. The $n$-adic concepts of an $n$-adic context $\left(K_{1}, \ldots, K_{n}, Y\right)$ are exactly the maximal $n$-tuples $\left(A_{1}, \ldots, A_{n}\right)$ in $2^{K_{1}} \times \cdots \times 2^{K_{n}}$, where $A_{1} \times \cdots \times A_{n} \subseteq Y$ with respect to component-wise set inclusion (Voutsadakis, 2002). The notion of $n$-adic concept lattice can be introduced similarly to the triadic case (Voutsadakis, 2002).

\subsection{Maximal closed connected sets}

Here we introduce necessary defintions and results from a series of papers on mining maximal closed connected sets (Spyropoulou et al., 2014, Lijffijt et al. 2016). Note that the authors prefer to use terminology close to relational databases but the main definitions can be easily reproduced in terms of $k$-partite graphs; to find related works in FCA community one may refer to Relational Concept Analysis Hacene et al. (2013).

Spyropoulou et al. (2014) formalised a multi-relational database (MRD) as a tuple $\mathbb{D}=(E, t, \mathcal{R}, R)$, where $E$ is a finite set of entities that is partitioned into $n$ entity types by a mapping $t: E \rightarrow\{1, \ldots, n\}$, i.e., $E=E_{1} \sqcup \cdots \sqcup E_{k}{ }^{2}$ with $E_{i}=\{e \in E \mid t(e)=i\}$. Moreover, $R \subseteq\{\{i, j\} \mid i, j \in\{1, \ldots, k\}, i \neq j\}$ is a set of relationship types such that for each $\{i, j\} \in R$ there is a binary relation $\mathcal{R}_{\{i, j\}} \subseteq\left\{\left\{e_{i}, e_{j}\right\} \mid e_{i} \in E_{i}, e_{j} \in E_{j}\right\}$. The set $\mathcal{R}$ then is the union of

\footnotetext{
${ }^{2}$ Here and later, $\sqcup$ means disjoint union.
} 
all these relations, i.e., $\mathcal{R}=\bigcup_{\{i, j\} \in R} \mathcal{R}_{\{i, j\}}$. This definition allows relationship types can be many-to-many, one-to-many, or one-to-one, depending on how many relationships the entities of either entity types can participate in. The authors do not allow relationship types between an entity type and itself since they mainly concentrate on relations between entities of different types, but the former can be modeled by having two copies of the same entity type and a relationship type between them.

Definition 1 (Completeness) (Spyropoulou et al., 2014) $A$ set $F \subseteq E$ is complete if for all $e, \tilde{e} \in F$ with $\{t(e), t(\tilde{e})\} \in R$ it holds that $\{e, \tilde{e}\} \subseteq \mathcal{R}_{\{t(e), t(\tilde{e})\}}$.

Definition 2 (Connectedness) (Spyropoulou et al., 2014) A set $F \subseteq E$ is connected if for all $e, \tilde{e} \in F$ there is a sequence $e=e_{1}, \ldots, e_{l}=\tilde{e}$ with $\left\{e_{1}, \ldots, e_{l}\right\} \subseteq$ $F$ such that for $i \in\{1, \ldots, l\}$ it holds that $\left\{e_{i}, e_{i+1}\right\} \in R$.

It implies that a subset of size larger than one can be connected only if it contains entities of at least two different types.

A set $F \subseteq E$ is a Complete Connected Subset (CCS) if it satisfies both connectedness and completeness.

A Maximal Complete Connected Subset (MCCS) is a CCS to which no element can be added without violating connectedness or completeness.

For a database $\mathbb{D}=(E, t, \mathcal{R}, R)$ the set system of CCSs, is defined as $\mathcal{F}_{\mathbb{D}}=$ $\{F \subseteq E \mid F$ is connected and complete $\}$. From an algorithmic point of view, the property of strong accessibility means that for two $\operatorname{CCSs} X, Y \in \mathcal{F}_{\mathbb{D}}$ with $X \subseteq Y$, it is possible to iteratively extend $X$ by one element at a time, only passing via sets from the set system and finally obtain $Y$. Formally, for a set system $F \subseteq 2^{A}$, where $A$ is the ground set, and a set $F \in \mathcal{F}$, let us denote by $\operatorname{Aug}(F)=\{a \in A \mid F \cup\{a\} \in \mathcal{F}\}$ the set of valid augmentation elements of $F$. Then $\mathcal{F}$ is called strongly accessible if for all $X \subset Y \subseteq A$ with $X, Y \in \mathcal{F}$ there is an element $e \in(A u g(X) \backslash X) \cap Y$.

Theorem 1 (Spyropoulou et al., 2014) For all relational databases $\mathbb{D}=(E, t, \mathcal{R}, R)$, the set system $\mathcal{F}_{\mathbb{D}}$ of CCSs is strongly accessible. 
Specifically for the set system $\mathcal{F}_{\mathbb{D}}$ of CCSs, and given a relational database $\mathbb{D}=(E, t, \mathcal{R}, R)$, the set $\operatorname{Aug}(F)$ corresponds to the following set: $\operatorname{Aug}(F)=$ $\{e \in E \mid F \cup\{e\}$ is complete and connected $\}$. Note that for the sake of efficiency $\operatorname{Aug}(F)$ can be recursively updated.

To define a closure operator for the set system $\mathcal{F}_{\mathbb{D}}$ the authors make use of the set of compatible entities which is defined as follows:

Definition 3 (Compatible entities) (Spyropoulou et al., 2014) For a relational database $\mathbb{D}=(E, t, \mathcal{R}, R)$ the set of compatible entities of a set $F \in \mathcal{F}_{\mathbb{D}}$ is defined as $\operatorname{Comp}(F)=\{e \in E \mid F \cup e$ is complete $\}$.

Definition 4 (g operator) (Spyropoulou et al. 2014) For a relational database $\mathbb{D}=(E, t, \mathcal{R}, R)$ the operator $g: \mathcal{F}_{\mathbb{D}} \rightarrow 2^{E}$ is defined as $g(F)=\{e \in \operatorname{Aug}(F) \mid$ $\operatorname{Comp}(F \cup e)=\operatorname{Comp}(F)\}$.

Proposition 1 (Spyropoulou et al., 2014) For all relational databases $\mathbb{D}=$ $(E, t, \mathcal{R}, R)$, the codomain of the $g$ operator is the set system $\mathcal{F}_{\mathbb{D}}$ of CCSs and $g$ is extensive and monotone.

Proposition 2 (Spyropoulou et al., 2014) For all relational databases $\mathbb{D}=$ $(E, t, \mathcal{R}, R)$ with the property that $e \in E$ such that $\{e\} \cup E_{i}$ is complete and connected for an $i \in t(E)$, the operator $g$ is idempotent.

Corollary 1 (Spyropoulou et al., 2014) For all relational databases $\mathbb{D}=(E, t, \mathcal{R}, R)$, with the property that $e \in E$ such that $\{e\} \cup E_{i}$ is complete and connected for an $i \in t(E)$, the operator $g$ is a closure operator.

Note that, the technical requirement in Proposition 2 for $g$ being idempotent may be fulfilled by adding an isolated vertex $\left\{e_{0}\right\}$ to $E_{i}$ for all $E_{i} \subseteq E$ and $e \in E \backslash E_{i}$, where $E_{i} \cup\{e\}$ is CCS.

Example 1 In Figure 1 , on the left one can see the violation of idempotency of $g(\cdot)$ since $g\left(\left\{r_{1}, r_{2}, p_{1}\right\}\right)=\left\{r_{1}, r_{2}, p_{1}, f\right\}$ and $g\left(g\left(\left\{r_{1}, r_{2}, p_{1}\right\}\right)\right)=\left\{r_{1}, r_{2}, p_{1}, f, u_{1}, u_{2}, u_{3}\right\}$. 

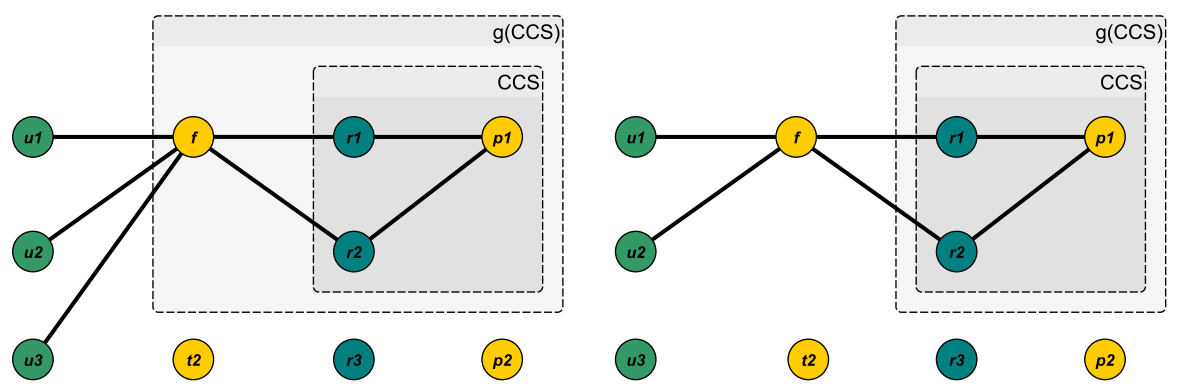

Figure 1: On idempotency of $g(\cdot)$

On the right graph of Figure 1 the idempotency fulfills since $g\left(\left\{r_{1}, r_{2}, p_{1}\right\}\right)=$ $g\left(g\left(\left\{r_{1}, r_{2}, p_{1}\right\}\right)\right)=\left\{r_{1}, r_{2}, p_{1}\right\}$. It happens since for the left graph

$$
\operatorname{Comp}\left(\left\{r_{1}, r_{2}, p_{1}\right\} \cup f\right)=\left\{r_{1}, r_{2}, p_{1}, f, u_{1}, u_{2}, u_{3}\right\}=\operatorname{Comp}\left(\left\{r_{1}, r_{2}, p_{1}\right\}\right),
$$

but for the right one

$\operatorname{Comp}\left(\left\{r_{1}, r_{2}, p_{1}\right\} \cup f\right)=\left\{r_{1}, r_{2}, p_{1}, f, u_{1}, u_{2}\right\} \neq \operatorname{Comp}\left(\left\{r_{1}, r_{2}, p_{1}\right\}\right)=\left\{r_{1}, r_{2}, p_{1}, f, u_{1}, u_{2}, u_{3}\right\}$.

\section{Pitfalls of recent candidates for closure operators in triadic case}

\subsection{Non-monotonicity of TriCons concept forming operator}

To simplify further considerations of tri-sets, triadic concepts and multirelational databases both as tuples and sets, we introduce two interrelated operators.

Definition 5 (Trabelsi et al., 2012) Let $\mathbb{K}=(G, M, B, I)$ be a formal tricontext. A triple $(X, Y, Z)$ is called a triset of $\mathbb{K}$ iff $X \times Y \times Z \subseteq I$.

Note that Cerf et al. (2009) define a triset of $\mathbb{K}$ differently: $X \times Y \times Z \in$ $2^{G} \times 2^{M} \times 2^{B}$. We keep the former definition to work with $h(\cdot)$ in the original setting (Trabelsi et al., 2012).

Note that according the definition of Cartesian product, if at least one of the sets $X, Y$ or $Z$ is $\emptyset$ (Simovici \& Djeraba, 2008), then $X \times Y \times Z=\emptyset$, so $\emptyset \subseteq I$ 
and $(X, Y, Z)$ is a triset. However trisets $(X, Y, \emptyset)$ and $(X, \emptyset, Z)$ have different structure even though $X \times Y \times \emptyset=X \times \emptyset \times Z=\emptyset \subseteq I$.

Definition 6 For a formal tricontext $\mathbb{K}=(G, M, B, I)$ and any triple $(X, Y, Z) \subseteq$ $2^{G} \times 2^{M} \times 2^{B}$ (e.g. triconcept) of $\mathbb{K}$ the operator flat : $2^{G} \times 2^{M} \times 2^{B} \rightarrow 2^{G \sqcup M \sqcup B}$ is defined as follows: $\operatorname{flat}(X, Y, Z)=X \sqcup Y \sqcup Z$.

Definition 7 For a given formal n-context $\mathbb{K}=\left(E_{1}, \ldots, E_{n}, I \subseteq E_{1} \times \ldots \times E_{n}\right)$ (or multi-relational database $\mathbb{D}=(E, t, \mathcal{R}, R)$ ), where $E=\operatorname{flat}\left(E_{1}, \ldots, E_{n}\right)$, and $S \subseteq 2^{E}$, the operator tuple : $2^{E} \rightarrow 2^{E_{1}} \times \ldots \times 2^{E_{n}}$ is defined as follows: tuple $(S)=\left(E_{1} \cap S, \ldots, E_{n} \cap S\right)$.

Triple compositions of tuple $(\cdot)$ and $f l a t(\cdot)$ operators form identity operators tuple $(f \operatorname{lat}(\operatorname{tuple}(\cdot)))=i d_{S}(\cdot)$ and flat(tuple $($ flat ()$\left.)\right)=i d_{T}(\cdot)$ over sets and tuples respectively.

Note that for trisets $t_{1}=\left(A_{1}, B_{1}, C_{1}\right)$ and $t_{2}=\left(A_{2}, B_{2}, C_{2}\right), t_{1} \sqsubseteq t_{2}$ means that $A_{1} \times B_{1} \times C_{1} \subseteq A_{2} \times B_{2} \times C_{2}$, i.e. every triple $(a, b, c) \in\left(A_{1}, B_{1}, C_{1}\right)$ is in $\left(A_{2}, B_{2}, C_{2}\right)$. It follows that $\sqsubseteq$ is not antisymmetric, since e.g. $(X, Y, \emptyset) \sqsubseteq$ $(X, \emptyset, Z)$ and $(X, \emptyset, Z) \sqsubseteq(X, Y, \emptyset)$, but $(X, Y, \emptyset) \neq(X, \emptyset, Z)$. Thus every preorder $\left(\mathcal{T} \subseteq 2^{G} \times 2^{M} \times 2^{B} \cap 2^{I}, \sqsubseteq\right)$ have all equivalence classes of cardinality 1 except $[\emptyset]=\{(\emptyset, \emptyset, \emptyset), \ldots,(G, \emptyset, \emptyset) \ldots,(\emptyset, M, B)\}$ of cardinality $2^{|G|+|M|+|B|}-1$.

Definition 8 (Trabelsi et al., 2012) Let $S=(X, Y, Z)$ be a tri-set of $\mathbb{K}=$ $(G, M, B, I \subseteq G \times M \times B)$. The mapping $h: 2^{G} \times 2^{M} \times 2^{B} \cap 2^{I} \rightarrow 2^{G} \times 2^{M} \times 2^{B}$ is defined as follows:

$$
\begin{aligned}
& h(S)=\{(U, V, W) \mid U=\{g \in G \mid \forall m \in Y, \forall b \in Z:(g, m, b) \in I\} \\
& \wedge V=\{m \in M \mid \forall g \in U, \forall b \in Z:(g, m, b) \in Y\} \wedge W=\{b \in B \mid \forall g \in \\
& U, \forall m \in V:(g, m, b) \in Y\}
\end{aligned}
$$

Note that every triconcept is a maximal or closed triset, i.e. a triset that cannot be extended by triples from $I$ being a triset.

Proposition $3 h(\cdot)$ is extensive and idempotent by $\sqsubseteq$ on $T=\{t \mid t$ is a triset of $\mathbb{K}\}=$ 
$\left\{(X, Y, Z) \in 2^{G} \times 2^{M} \times 2^{B} \mid(X, Y, Z) \subseteq I\right\}$ and every fixpoint $f$ of $h$ (i.e. $h(f)=f)$ is a triconcept of $\mathbb{K}$.

Proof. One can find the proof of extensivity and idempotency in (Trabelsi et al., 2012). It is easy to see that every formal triconcept is a fixpoint of $h(\cdot)$ and every triset $(X, Y, Z)$ is transformed by $h(\cdot)$ to the triconcept $((Y \times$ $Z)^{(1)},\left((Y \times Z)^{(1)} \times Z\right)^{(2)},\left((Y \times Z)^{(1)} \times\left((Y \times Z)^{(1)} \times Z\right)^{(2)}\right)^{(3)}$. Indeed, all formal triconcepts should be listed since a triset is allowed to be a triple with at least one component being $\emptyset$.

Theorem 2 For a given tricontext $\mathbb{K}=(G, M, B, I \subseteq G \times M \times B)$ and its associated triset system $\mathcal{T}=\left\{(X, Y, Z) \in 2^{G} \times 2^{M} \times 2^{B} \mid(X, Y, Z) \subseteq I\right\}$ operator $h$ is not monotone w.r.t. $\sqsubseteq$.

Proof. To construct a violating example, one needs two different triconcepts with the same extent, $c_{1}=\left(X, Y_{1}, Z_{1}\right)$ and $c_{2}=\left(X, Y_{2}, Z_{2}\right)$ of $\mathbb{K}$ such that $Y_{1} \subset Y_{2}$ and $Z_{1} \supset Z_{2}$.

Consider the tri-set $s=\left(X, Y_{1}, Z_{2}\right)$ :

$$
s \sqsubseteq c_{1} \Rightarrow h(s)=c_{2} \nsubseteq h\left(c_{1}\right)=c_{1}
$$

Example 2 For the tricontext in Figure 2, the violating example for monotonicity of $h(\cdot)$ is as follows:

$$
\begin{gathered}
x=\left(\left\{u_{1}, u_{2}\right\},\left\{t_{1}\right\},\left\{r_{1}\right\}\right) \sqsubseteq y=\left(\left\{u_{1}, u_{2}\right\},\left\{t_{1}\right\},\left\{r_{1}, r_{2}\right\}\right) \Rightarrow \\
h(x))=\left(\left\{u_{1}, u_{2}\right\},\left\{t_{1}, t_{2}\right\},\left\{r_{1}\right\}\right) \nsubseteq h(y)=\left(\left\{u_{1}, u_{2}\right\},\left\{t_{1}\right\},\left\{r_{1}, r_{2}\right\}\right) .
\end{gathered}
$$

Definition 9 (Ganter \& Wille (1999), p.237, Ganter \& Glodeanu (2012)) A relation $R \subseteq G \times M$ is called a Ferrers relation iff there are subsets $A_{1} \subset A_{2} \subset$ $A_{3} \ldots \subseteq G$ and $M \supseteq B_{1} \supset B_{2} \supset B_{3} \supset \ldots$ such that $R=\bigcup_{i} A_{i} \times B_{i}$.

$R$ is called a Ferrers relation of concepts of $(G, M, I)$ iff there are formal concepts $\left(A_{1}, B_{1}\right) \leq\left(A_{2}, B_{2}\right) \leq\left(A_{3}, B_{3}\right) \leq \ldots$ such that $R=\bigcup_{i} A_{i} \times B_{i}$. 


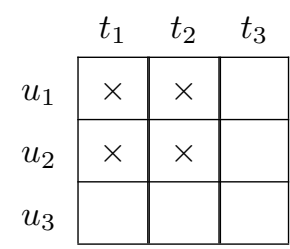

$r_{1}$

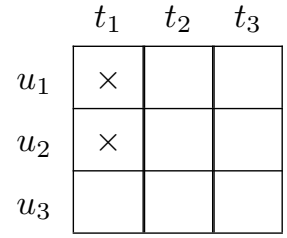

$r_{2}$

Figure 2: A small example with Bibsonomy data

Proposition 4 (Ganter \& Glodeanu, 2012) Any Ferrers relation $R \subseteq I$ is contained in a Ferrers relation of concepts of $(G, M, I)$.

Corollary 2 Let $\mathbb{K}=(G, M, B, I)$ be a formal tricontext, and $\mathbb{K}_{X}^{M B}=\left(M, B, I_{X}\right)$ such that $(m, b) \in I_{X}$ iff $(g, m, b) \in I \cap X \times M \times B$, and $I_{X}$ be Ferrers relation of concepts of $\mathbb{K}_{X}^{M B}$. Operator $h$ is not monotone for every pair of trisets $(X, Y, Z)$ and $\left(X, Y_{i}, Z_{i}\right)$ such that $Y \subseteq Y_{i}, Z \subseteq Z_{j}$, and $\left(Y_{i}, Z_{i}\right) \leq\left(Y_{j}, Z_{j}\right)$ are concepts of $\mathbb{K}_{X}^{M B}$.

\subsection{Inconsistency of MCCS closure}

\subsubsection{Lossy hyperedge encoding and phantom edges}

In case of $k$-partite graph encoding we can meet information loss in a form of new hyperedges. Below we provide this encoding from polyadic contexts to multi-relation databases with $n$ types of entities.

Let $\mathbb{K}=\left(K_{1}, \ldots, K_{n}, I\right)$ be a polyadic formal context, then $\mathbb{D}=(E=$ $\left.K_{1} \sqcup \ldots \sqcup K_{n}, t, \mathcal{R}, R\right)$ be the corresponding multi-relation database, where $t$ maps entities from $E$ into their types from 1 to $n, R=\{\{i, j\} \mid i, j \in\{1, \ldots, n\}, i \neq j\}$ and $\mathcal{R}=\bigcup_{\{i, j\} \in R} \mathcal{R}_{\{i, j\}}$ for the binary relations $\mathcal{R}_{\{i, j\}}=\left\{\left\{e_{i}, e_{j}\right\} \mid e_{i} \in\right.$ $K_{i}, e_{j} \in K_{j}$ and $e_{i}, e_{j}$ are related by $\left.I\right\}$.

Example 3 Imagine that we have three hyperedges $\left\{u, t, r_{0}\right\},\left\{u, t_{0}, r\right\},\left\{u_{0}, t, r\right\}$, and then encode them as edges in a 3-partite graph, we obtain

$$
\{u, t\},\left\{u, r_{0}\right\},\left\{t, r_{0}\right\},\left\{t_{0}, r\right\},\{u, r\},\left\{u, t_{0}\right\},\{t, r\},\left\{u_{0}, r\right\} \text {, and }\left\{u_{0}, t\right\} \text {. }
$$


Since we now have $\{u, t\},\{u, r\}$, and $\{t, r\}$ in our graph, we should inevitably decode a new hyperedge, $\{u, t, r\}$. See Figure 3 .

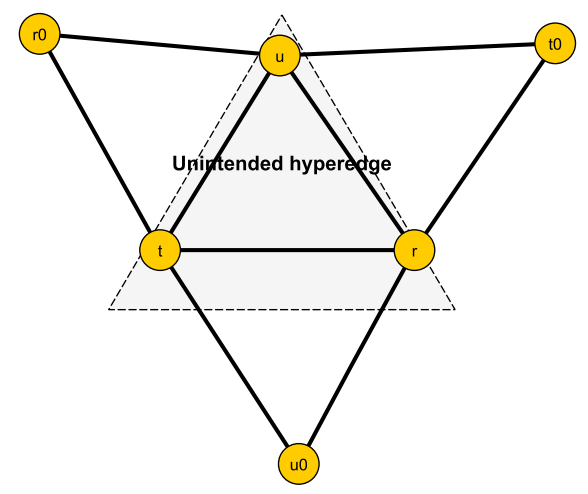

Figure 3: A phantom hyperedge as a structure loss

Taking the last fact into account and following the definition of MCCS or applying $g(\cdot)$ to respective CCS, we should obtain in general case a different or extra pattern(s) in addition to triconcepts in $k$-partite encoding. Thus in Example 3 there are three MCCSs, $\left\{u, t_{0}, t, r\right\},\left\{u, u_{0}, t, r\right\}$, and $\left\{u, t, r_{0}, r\right\}$, that are different from set representation of formal triconcepts, $\left\{u, t_{0}, r\right\},\left\{u_{0}, t, r\right\}$, $\left\{u, t, r_{0}\right\}$, of the initial tricontext, respectively.

\subsubsection{Closed but non-maximal patterns}

As one can see from the example in Table 1, the technical condition for idempotency of $g(\cdot)$ is fulfilled. The corresponding tripartite graph is depicted in Figure 4 .

However for the CCS pattern $X=\left\{u_{1}, u_{2}, t_{1}, r_{1}\right\}$ the result of $g(X)$ coincides with $X$ but it is not maximal. Indeed, there exist two maximal closed and connected patterns corresponding to triconcepts, $X \cup t_{2}=\left\{u_{1}, u_{2}, t_{1}, t_{2}, r_{1}\right\}$ and $X \cup r_{2}=\left\{u_{1}, u_{2}, t_{1}, r_{1}, r_{2}\right\}$.

It is so, since $\operatorname{Comp}(X)=X \cup\left\{t_{2}, r_{2}\right\}$, but $\operatorname{Comp}\left(X \cup t_{2}\right)=X \cup t_{2}$ and $\operatorname{Comp}\left(X \cup r_{2}\right)=X \cup r_{2}$. 
Table 1: A small example with Bibsonomy data

\begin{tabular}{c|c|c|c|}
\multicolumn{1}{c}{} & \multicolumn{1}{c}{$t_{1}$} & \multicolumn{1}{c}{$t_{2}$} & \multicolumn{1}{c}{$t_{3}$} \\
\cline { 2 - 4 }$u_{1}$ & $\times$ & $\times$ & \\
\cline { 2 - 4 }$u_{2}$ & $\times$ & $\times$ & \\
\cline { 2 - 4 }$u_{3}$ & & & \\
\hline
\end{tabular}

$r_{1}$

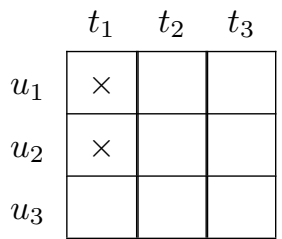

$r_{2}$

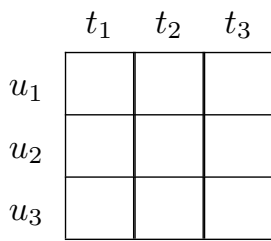

$r_{3}$

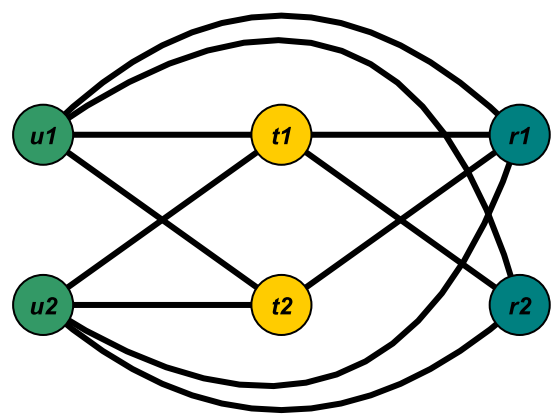

(43)

(t3

Figure 4: A counter example: closed but non-maximal patterns

Proposition 5 Let $\mathcal{F}_{\mathbb{D}}$ be a $C C S$ system and $\mathcal{H} \subseteq \mathcal{F}_{\mathbb{D}}$ such that $|\mathcal{H}| \geq 2$, every $H \in \mathcal{H}$ is maximal and there exists a CCS $X=\bigcap_{H \in \mathcal{H}} H \neq \emptyset$, then $g(X)=X$ but $X$ is not an MCCS.

Proof. Since there exist more than two MCCSs $H_{i}, H_{j} \in H$, we obtain $X \subset H_{i}$ and $X \subset H_{j}$. Therefore $H_{i} \backslash X \subseteq \operatorname{Comp}(X)$ and $H_{j} \backslash X \subseteq \operatorname{Comp}(X)$. However for $h_{i} \in H_{i}$ and $h_{j} \in H_{j}, \operatorname{Comp}\left(X \cup h_{i}\right) \neq \operatorname{Comp}\left(X \cup h_{j}\right)$ since otherwise it violates maximality of $H_{i}$.

Let us introduce generalised Ferres relation of $n$-concepts (for 3 -adic case see (Glodeanu, 2013)) .

Definition $10 A$ relation $R \subseteq K_{1} \times \cdots \times K_{n}$ is called a generalised Ferrers relation iff $\exists j \in\{1, \ldots, n\} \forall i \in\{1, \ldots, n\} \backslash\{j\} A_{1 i} \subset A_{2 i} \subset A_{3 i} \ldots \subseteq K_{i}$, and 
$K_{j} \supseteq A_{1 j} \supset A_{2 j} \supset A_{3 j} \supset \ldots$ such that $R=\bigcup_{k} A_{k 1} \times \ldots \times A_{k n}$.

$R$ is called a Ferrers relation of $n$-concepts of $\left(K_{1}, \ldots, K_{n}, I\right)$ iff there are formal $n$-concepts $\left(A_{11}, \ldots, A_{1 n}\right) \lesssim_{k}\left(A_{21}, \ldots, A_{2 n}\right) \lesssim_{k}\left(A_{31}, \ldots, A_{3 n}\right) \lesssim_{k} \ldots$ such that $R=\bigcup_{i} A_{i 1} \times \ldots \times A_{i n}$.

Corollary 3 Let $\mathbb{K}=\left(K_{1}, \ldots, K_{n}, I\right)$ be a polyadic formal context such that $I$ is a Ferrers relation of $n$-concepts and $\mathbb{D}=\left(E=K_{1} \sqcup \ldots \sqcup K_{n}, t, \mathcal{R}, R\right)$ be the corresponding multi-relation database. Operator $g$ does not produce an MCCS for flat $\left(A_{j 1} \cap A_{i 1}, \ldots, A_{j n} \cap A_{i n}\right)$ obtained from any pair of concepts of $\mathbb{K},\left(A_{i 1}, \ldots, A_{i n}\right) \lesssim_{k}\left(A_{j 1}, \ldots, A_{j n}\right)$, where $A_{i} s \neq A_{j} s, s, k \in\{1, \ldots, n\}$ and $s \neq k$.

\section{Closure operator for triconcepts}

There are $n$-contexts, where $h(\cdot)$ is not a closure that results in formal concepts because of non-idempotency and closure operator $g(\cdot)$ produces CCSs that are not necessary maximal, e.g. caused by non-uniqueness of possible extensions of input patterns. Moreover, the lossy data encoding by $n$-partite graph instead of $n$-partite hypergraph results in phantom $n$-adic edges and extra elements in resulting patterns.

So, to overcome the difficulty at least for generation of $n$-concepts we may adjust the set systems such that $h(\cdot)$ and $g(\cdot)$ could operate. Informally, we need to weed all patterns or phantom hyperedges that result in undesirable behaviour of $h(\cdot)$ and $g(\cdot)$, the candidates to closure operators.

Definition 11 Let $\mathbb{K}=\left(K_{1}, K_{2}, K_{3}, I\right)$ be a triadic formal context. A triset $S$ is called a (maximal) switching generator of the context $\mathbb{K}$ iff $S=$ tuple $\left(\right.$ flat $\left(c_{1}\right) \cap$ flat $\left.\left(c_{2}\right)\right) \neq \emptyset$, where $c_{1}$ and $c_{2}$ are concepts of $\mathbb{K}$.

Theorem 3 Let $\mathbb{K}=\left(K_{1}, K_{2}, K_{3}, I\right)$ be a triadic formal context. The set system $\mathcal{F}_{\mathbb{K} \ominus \mathcal{S}}=\mathcal{T} \backslash \mathcal{S}$ is a correct set system for formal triconcept generation by $h(\cdot)$ in $\mathbb{K}$, where $\mathcal{T}=\left\{(X, Y, Z) \in 2^{G} \times 2^{M} \times 2^{B} \mid(X, Y, Z) \subseteq I\right\}$ and $\mathcal{S}=\{S \mid S$ is a switching generator of $\mathbb{K}\}$. 
Proof. Since there is no a switching generator in $\mathcal{F}_{\mathbb{K} \ominus \mathcal{S}}$, monotony of $h(\cdot)$ is fulfilled.

Assume that monotony is violated by trisets $x$ and $y$, that is $x \sqsubseteq y \rightarrow$ $h(x) \nsubseteq h(y)$. By extensivity of $h(\cdot)$ and transitivity of $\sqsubseteq$, it implies $x \sqsubseteq h(x)$ and $x \sqsubseteq h(y)$. Hence, $x \sqsubseteq \operatorname{tuple}(\operatorname{flat}(h(x)) \cap \operatorname{flat}(y))$, i.e. $x$ is a switching generator. Contradiction.

Since every formal triconcept is not a switching generator, none of triconcepts has been deleted from $\mathcal{F}_{\mathbb{K} \ominus \mathcal{S}}$.

As for phantom triadic edges, unfortunately it is not possible to delete them from $\mathcal{R}$ since each phantom triadic edge $\left\{e_{i}, e_{j}, e_{k}\right\}$ is composed by $\left\{e_{i}, e_{j}\right\}$, $\left\{e_{j}, e_{k}\right\}$, and $\left\{e_{k}, e_{i}\right\}$, which are parts of "real" triadic hyperedges.

Let $\mathbb{K}$ be a formal tricontext and $\mathbb{D}$ be the corresponding multi-relational database, $\mathcal{P}=\left\{\operatorname{tuple}(e) \mid e=\left\{e_{i}, e_{j}, e_{k}\right\}\right.$ is a phantom edge in $\left.\mathcal{R}\right\}$ then a test whether an MCCS forms triset can be done as follows:

1. For an MCCS $s$ form tuple $(s)=(X, Y, Z)$;

2. Check whether $t=X \times Y \times Z \backslash e$ forms a triset of $\mathbb{K}$, where $e \in \mathcal{P}$;

3. If yes, then output $t$;

4. Delete $s$ from the output otherwise.

To make sure that $t$ is a triconcept, one need to check $h(t)=t$.

Since traditionally closure operators were introduced for partial orders over set inclusion, we would like to avoid dealing with preoder $\sqsubseteq$ over trisets and work with set inclusion of their set representations instead.

For tricontext $\mathbb{K}=\left(K_{1}, K_{2}, K_{3}, I\right)$ we consider a family of operators

$$
\begin{gathered}
\left\{\sigma_{i j k} \mid \sigma_{i j k}: 2^{K_{1}} \times 2^{K_{2}} \times 2^{K_{3}} \rightarrow 2^{K_{1}} \times 2^{K_{2}} \times 2^{K_{3}}\right. \text { such that } \\
\sigma_{i j k}:\left(X_{1}, X_{2}, X_{3}\right) \mapsto\left(Y_{1}, Y_{2}, Y_{3}\right), \text { where } \\
\left.Y_{i}=\left(X_{j} \times X_{k}\right)^{(i)}, Y_{j}=\left(Y_{i} \times X_{k}\right)^{(j)}, Y_{k}=\left(Y_{i} \times Y_{j}\right)^{(k)} \text {, where }\{i, j, k\}=\{1,2,3\}\right\} .
\end{gathered}
$$

The cardinality of the family is $3 !=6$ and $n$ ! for its n-ary case generalisation. 
Proposition 6 Operators $\sigma_{i j k}(\cdot)$ are not commutative, i.e. $\sigma_{i j k}\left(\sigma_{l m n}(\cdot)\right) \neq$ $\sigma_{l m n}\left(\sigma_{i j k}(\cdot)\right)$, where $(i, j, k) \neq(l, m, n)$ and $\{i, j, k\}=\{1,2,3\}$.

Proof. Consider a tricontext given below.

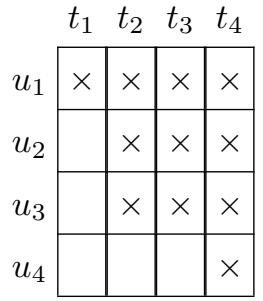

$r_{1}$

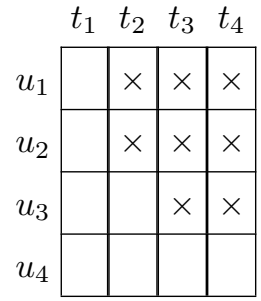

$r_{2}$

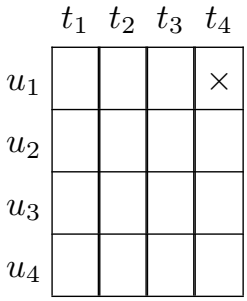

$r_{3}$

The system of all switching generators $\mathcal{S}$ contains $s_{1}=\left\{u_{1}, t_{4}, r_{1}\right\}$ and $s_{2}=$ $\left\{u_{1}, u_{2}, t_{3}, t_{4}, r_{1}\right\}$.

$s_{1}$ proves that $\sigma_{i_{--}}(\cdot) \neq \sigma_{j_{--}}(\cdot) \neq \sigma_{k_{--}}(\cdot)$ and

$s_{2}$ proves that $\sigma_{i j k}(\cdot) \neq \sigma_{i k j}(\cdot)$ for $\{i, j, k\}=\{1,2,3\}$.

The fact that $\sigma_{l m n} \sigma_{i j k}(\cdot)=\sigma_{i j k}(\cdot)$ proves the proposition.

Theorem 4 For $\mathbb{K}=\left(K_{1}, K_{2}, K_{3}, I\right)$ and the associated triset system $\mathcal{T}$ there is no an associated closure operator in case there exist at least two concepts $c_{1}=\left(X_{1}, Y_{1}, Z_{1}\right)$ and $c_{2}=\left(X_{1}, Y_{2}, Z_{2}\right)$ such that they have the common nonempty maximal switching generator $s$, i.e. tuple $\left(f l a t\left(c_{1}\right) \cap \operatorname{flat}\left(c_{2}\right)\right) \neq \emptyset$.

Proof. Let $\sigma$ be a closure operator for $\mathbb{K}$. Since $s \sqsubset c_{1}$ and $s \sqsubset c_{2}$ then $\sigma(s)$ should result in $c_{i}$ which is either $c_{1}$ or $c_{2}$ (or one of other concepts $c_{k}$ with $s \sqsubset c_{k}$ if any exist). So let $\sigma(s)=c_{i}$ and consider $s \sqsubseteq c_{j}$; it implies that $\sigma(s)=c_{i} \nsubseteq \sigma\left(c_{j}\right)=c_{j}$ for $i \neq j$, and $\{i, j\}=\{1,2\}$. Contradiction.

As it has been shown, $\mathcal{F}_{\mathbb{K} \ominus S}$ is a correct set system for $h(\cdot)=\sigma_{123}(\cdot)$ being a closure operator. It is easy to see that this system is correct for $\sigma_{i j k}(\cdot)$.

To summarise properties of $\mathcal{F}_{\mathbb{K} \ominus S}$ and show its difference from set systems in (Boley et al. 2010; Spyropoulou et al., 2014) we recall the following properties of set systems.

Definition 12 A non-empty set system $(E, \mathcal{F})$ is called

1. accessible if for all $X \in \mathcal{F} \backslash\{\emptyset\}$ there is an $e \in X$ such that $X \backslash\{e\} \in \mathcal{F}$, 
2. an independence system if $Y \in F$ and $X \subseteq Y$ together imply $X \in F$,

3. confluent if for all $I, X, Y \in \mathcal{F}$ with $\emptyset \in I \subseteq X$ and $I \subseteq Y$ it holds that $X \cup Y \in F$.

4. strongly accessible if it is accessible and for all $X, Y \in F$ with $X \subset Y$, there is an $e \in Y \backslash X$ such that $X \cup\{e\} \in \mathcal{F}$.

Proposition 7 1) Set system $\mathcal{F}_{\mathbb{K} \ominus S}$ of all sets that form trisets is accessible and 2) not independent. 3) It is not a closure system. 4) It is confluent. 5) It is strongly accessible.

Proof. 1. Every set of $\mathcal{F}_{\mathbb{K} \ominus S}$ forms a triset $t$. Even if it contains some switching generator $s$, we can then remove any $e \in \operatorname{flat}(s)$ from $t$, the resulting set flat $(t) \backslash e$ is in $\mathcal{F}_{\mathbb{K} \ominus S}$ (switching generator free system) since it is a triset and contains at least one element not included in a switching generator. Empty set (or empty set of triples) is not in $\mathcal{F}_{\mathbb{K} \ominus S}$ because it is a universal switching generator.

2. Since some concepts may contain switching generators by triset set inclusion, it implies that these switching generators are not $\mathcal{F}_{\mathbb{K} \ominus S}$.

3. On the contrary, every pair of concepts $X, Y \in \mathcal{F}_{\mathbb{K} \ominus S}$ implies that $X \cap Y \notin$ $\mathcal{F}$ (anti-sharing).

4. Since there is no such non-empty $I \in \mathcal{F}_{\mathbb{K} \ominus S}$ being a triset of two different concepts it trivially holds.

5. If $X \subset Y$ for $X, Y \in \mathcal{F}_{\mathbb{K} \ominus S}, X$ does not form a formal concept (because of antiordinal relations) or switching generator. So adding any element $e$ from $Y \backslash X$ leaves $X \cup\{e\}$ being a triset.

A detailed study of algorithmic issues is out of scope the paper, however, Boley et al. (2010) reported a simple algorithm for Problem 1, i.e. listing of all fixed points of a partially defined closure operator, which is correct for strongly accessible set systems.

Problem 1 (list-closed-sets) Given a set system $(E, \mathcal{F})$ with $\emptyset \in \mathcal{F}$ and a closure operator $\sigma: \mathcal{F} \rightarrow \mathcal{F}$, list the elements of $\sigma(\mathcal{F})=\{F \mid F \in \mathcal{F}: \sigma(F)=F\}$. 
It is questionable whether the weeding step can be efficiently incorporated into closure listing algorithm. Thus, in the worst case, i.e. for power tricontext $\mathbb{K}=(\{1 \ldots k\},\{1 \ldots k\},\{1 \ldots k\}, \neq)$, the number of triconcepts equals $3^{k}$ (Biedermann, 1998). The number of switching generators is greater than that of the concepts of $\mathbb{K}$ for $k>2$ and not polynomial as given in Theorem 5

Theorem 5 For a power tricontext $\mathbb{K}=(\{1 \ldots k\},\{1 \ldots k\},\{1 \ldots k\}, \neq)$ the number of switching generators is $4^{k}-3^{k}$.

Proof. One can prove the theorem by direct calculation of the triple sum below:

$$
\sum_{k_{1}=0}^{k-1} \sum_{k_{2}=0}^{k-k_{1}-1} \sum_{k_{3}=0}^{k-k_{1}-k_{2}-1} C_{k}^{k_{1}} C_{k-k_{1}}^{k_{2}} C_{k-k_{1}-k_{2}}^{k_{3}}
$$

Theorems 34 and propositions 76 can be generalised for $n$-ary case in a similar way. For example, the general version of Theorem 5 is provided as Theorem 6 .

Theorem 6 For a power polyadic $n$-context $\mathbb{K}=(\{1 \ldots k\}, \ldots,\{1 \ldots k\}, \neq)$ the number of switching generators is $(n+1)^{k}-n^{k}$.

In Kuznetsov (2004), the complexity of the problem "Number of all concepts" was addressed. Thus this problem is \#P-complete. Theorem 7 provides a similar justification, showing that the problem "Number of all (maximal) switching generators" is intractable. To avoid complex technicalities we prove the theorem for $n=2$.

Theorem 7 The following problem "Number of all (maximal) switching generators" is \#P-complete:

Input: Context $\mathbb{K}=(G, M, I)$

Output: The number of all (maximal) switching generators of the context $\mathbb{K}$, i.e. $|\mathcal{S}|$. 
Proof. We reduce the following \#P-complete problem to ours: "The number of binary vectors that satisfy monotone 2 -CNF of the form $C=\bigwedge_{i=1}^{s}\left(x_{i, 1} \vee x_{i, 2}\right)$ ":

Input: Monotone (without negation) CNF with two variables in each disjunction $C=\bigwedge_{i=1}^{s}\left(x_{i, 1} \vee x_{i, 2}\right)$, where $x_{i, 1}, x_{i, 2} \in X=\left\{x_{1}, \ldots, x_{k}\right\}$ for all $i=\overline{1, s}$.

Output: Number of binary $k$-vectors (corresponding to the values of variables) that satisfy $\mathrm{CNF} C$.

First, we construct 2-DNF $D$, the negation of $C: \bigvee_{i=1}^{s}\left(\bar{x}_{i, 1} \wedge \bar{x}_{i, 2}\right)$,. Each conjunction is denoted $D_{i}=\left(\bar{x}_{i, 1} \wedge \bar{x}_{i, 2}\right), i=\overline{1, s}$. The set of binary vectors that satisfy $D$ is a union of the sets of binary vectors that satisfy a certain $D_{i}$. Each disjunction is satisfied by every binary $k$-vector with $k-2$ ones and two zeros in $i_{1}$-th and $i_{2}$-th components.

We reduce this problem to that of the number of switching generators by constructing the following context $\mathbb{K}=(G, M, I)$. The set of attributes is $M=\left\{m_{1}, \ldots, m_{k}\right\} \cup \bigcup_{i=1}^{s}\left\{m^{i, k-1}, m^{i, k}\right\}$, where elements of $\tilde{M}=\left\{m_{1}, \ldots, m_{k}\right\}$ are in one-to-one correspondence with variables from $X$. For some conjunction $D_{i}, i=\overline{1, s}$, we construct a context $\mathbb{K}_{i}=\left(G_{i}, M_{i}, I_{i}\right)$, where the set of attributes is $M_{i}=\tilde{M} \backslash\left\{m_{i, 1}, m_{i, 2}\right\} \cup\left\{m^{i, k-1}, m^{i, k}\right\}:=\left\{m^{i, 1}, \ldots, m^{i, k}\right\}$, the set of objects is $G_{i}=\left\{g_{i}^{0}, g_{i}^{1}, \ldots, g_{i}^{k-2}, g_{i}^{k-1}, \ldots, g_{i}^{2 k-2}\right\}$, and the relation $I_{i} \subseteq M_{i} \times G_{i}$ is defined as follows: $\left\{g_{i}^{0}\right\}^{\prime}=M_{i} \backslash\left\{m^{i, k}\right\},\left\{g_{i}^{j}\right\}^{\prime}=M_{i} \backslash\left\{m^{i, j}, m^{i, k}\right\}$ for $j=\overline{1, k-2},\left\{g_{i}^{j}\right\}^{\prime}=$ $M_{i} \backslash\left\{m^{i, j}, m^{i, k-1}, m^{i, k}\right\}$ for $j=\overline{k-1,2 k-4},\left\{g_{i}^{k-3}\right\}^{\prime}=M_{i} \backslash\left\{m^{i, k-1}, m^{i, k}\right\}$, and $\left\{g_{i}^{2 k-2}\right\}^{\prime}=\left\{m^{i, k-1}, m^{i, k}\right\}$. The context $\mathbb{K}$ is constructed as follows: $\mathbb{K}=$ $\left(\bigsqcup_{i=1}^{s} G_{i}, M, \bigsqcup_{i=1}^{s} I_{i}\right)$ with only one $\left\{g_{i}^{2 k-2}\right\} \in G_{i}$ (the remaining $G_{j}$-s do not contain $\left.\left\{g_{j}^{2 k-2}\right\}\right)$.

First, we show that every switching generator of $\mathbb{K}$ corresponds to a $k$-vector that satisfies $D$. Every switching generator of $\mathbb{K}$ is a switching generator of $\mathbb{K}_{i}$ for some $i$, which can be not unique. It is easy to see that intents of the context $\mathbb{K}_{i}$ form the power set of $M_{i}$, denoted by $2^{M_{i}}$. Elements of $2^{\tilde{M}}$ are in one-toone correspondence with binary $n$-vectors, where components are in one-to-one correspondence with elements of $M$ with the same number. Since for every nonempty $S \subseteq \tilde{M} \backslash\left\{m_{i, 1}, m_{i, 2}\right\}$ there are concepts $\left(S^{\prime}, S\right)$ and $\left(\left(S \cup m^{i, k-1}\right)^{\prime}, S \cup\right.$ 
$\left.m^{i, k-1}\right)$, their switching generator is $\left(\left(S \cup m^{i, k-1}\right)^{\prime}, S\right)$. A vector of this form satisfies $D_{i}$, since it has zeros at $i_{1}$-th and $i_{2}$-th places. Therefore, this vector satisfies $D$. To prove that the switching generator is provided when $S=\emptyset$, one may check that $\left(\left\{g_{i}^{2 k-2}\right\},\left\{m^{i, k-1}\right\}\right)$ is the switching generator of concepts $\left(\left\{g_{i}^{2 k-2}\right\},\left\{m^{i, k-1}, m^{i, k}\right\}\right)$ and $\left(\left\{m^{i, k-1}\right\}^{\prime},\left\{m^{i, k-1}\right\}\right)$.

It remains to show that binary $k$-vectors that satisfy $D$ are in one-to-one correspondence with intents of $\mathbb{K}$. In fact, each binary $k$-vector $v$ that satisfies $D$, satisfies $D_{i}$ for some $i$ (this $i$ may be not unique). Then this vector has zero $i_{1}$-th and $i_{2}$-th components. Therefore, the corresponding set of attributes $A$ belongs to $\tilde{M} \backslash\left\{m_{i, 1}, m_{i, 2}\right\} \subseteq 2^{M_{i}}$. If $A \neq \emptyset$, then concepts $\left(A^{\prime}, A\right)$ and $\left(\left(A \cup m^{i, k-1}\right)^{\prime}, A \cup m^{i, k-1}\right)$ has switching generator is $\left(\left(A \cup m^{i, k-1}\right)^{\prime}, A\right)$. If $A=\emptyset$, then there is a unique switching generator $\left(\left\{g_{i}^{2 k-2}\right\},\left\{m^{i, k-1}\right\}\right)$ for some $G_{i}$.

The one-to-one correspondence between the switching generators of concepts of $\mathbb{K}$ and binary $k$-vectors satisfying $D$ is realised. Thus, if we know the number of all switching generators of concepts of $\mathbb{K}$, we obtain the number of all vectors satisfying $D$ and, therefore, that of the vectors satisfying $C$. The reduction is polynomial in the input size, since the context $\mathbb{K}$ has $|M|=k+2 s$ attributes and $|G|=s(2 k-2)+1$ objects.

Similar theorem can be proved for $n=3$.

Corollary 4 The problem "Number of all (maximal) switching generators for $n=3 "$ is \#P-complete:

Input: Context $\mathbb{K}=(G, M, B, Y)$

Output: The number of all (maximal) switching generators of the context $\mathbb{K}$, i.e. $|\mathcal{S}|$.

The proof can be done in a similar way to the dyadic case, where $\mathbb{K}_{i}=$ $\left(G_{i}, M, B_{i}, Y_{i}\right)$ for each conjunction $D_{i}$ should have $B_{i}=\left\{b_{i}^{1}, b_{i}^{2}\right\}$ (in fact, it plays a role of $\left\{m^{i, k-1}, m^{i, k}\right\}$ from Theorem 7 such that each $A$ will result in two triconcepts $\left(U, A,\left\{b_{i}^{1}, b_{i}^{2}\right\}\right)$ and $\left(V, A,\left\{b_{i}^{1}\right\}\right), U \subseteq V$ with their maximal switching generator $\left(U, A,\left\{b_{i}^{1}\right\}\right)$. 


\section{Related work}

In fact, one of the first methods for triconept enumeration was TRIPAT (Krolak-Schwerdt et al., 1994) adopted Ganter's Next Closure algorithm in a nested manner for two-adic contexts generated from an input tricontext; it had been done even before the first formal treatment of 3FCA by Lehmann \& Wille (1995). This idea has been incarnated later in TRIAS for triconcepts enumeration with component-wise size constraints (Jäschke et al., 2006).

Due to intrinsic complexity of exhaustive enumeration of triconcepts and closed $n$-sets, the research focus has shifted to constrained pattern mining and searching for different relaxations. Thus, after the release of DATAPeELER (Cerf et al. 2009), the Fenster algorithm for faul-tolerant pattern discovery has been proposed (Cerf et al., 2013); the latter includes closed $n$-set mining as a particular case, allowing not all tuples inside dense $n$-sets to be present. Another approach, is the so-called OAC-triclustering for mining dense trisets (Ignatov et al., 2015) results in no more patterns than the number of tuples in an input relation having a fruitful property of containment of all triconcepts for a given tricontext within the resulting collection of trisets w.r.t. to componentwise inclusion under a properly selected minimal density constraint. A different approximation of triconcept can be realised within least square error minimisation criterion (see TriBox, Mirkin \& Kramarenko (2011)), which lead to a density-based pattern quality measure, namely the squared density of a triset (in sense of (Cerf et al., 2009) ) multiplied by its size, thus, expressing trade-off between the high number of non-missing tuples inside and the large size.

One more direction is to use factorisation to select only a(n) (optimal) subset of triconcepts, which are factors to decompose an input three-way Boolean tensor (Glodeanu, 2013: Belohlávek et al., 2013). Closed sets are helpful for mining numeric contexts as well; thus, Kaytoue et al. (2013) used 3FCA for searching maximal inclusion biclusters of constant values by treatment of attribute values as conditions. Spyropoulou et al. (2014) proposed MCCS patterns and the associated closure operator for $n$-partite graphs working with multi- 
relational data. They also performed experimental comparison their RMineR with DATAPEELER, which is not fully correct since $n$-ry relations being encoded as $n$-partite graphs result in phantom edges. Note that, in FCA domain, there is Relational Concept Analysis devoted to treatment of multi-relational data (Hacene et al., 2013). The group that works on MCCSs has recently proposed Complete Connected Proper Subsets (CCPS) to deal with relational data with structured attributes (Lijffijt et al., 2016), i.e. attributes with ordered values like real numbers, geographic location, time intervals, etc. Note that in FCA domain, to deal with data of complex description the so called Pattern Structures were proposed more than decade ago by Ganter \& Kuznetsov (2001) and found many succesfull applications (Kaytoue et al., 2015).

There is an interesting connection between biclique operators, their associated graphs (Crespelle et al., 2015), and switching generators; in these graphs, two vertices (maximal biclques) are connected if they have a non-empty intersection, which, under some conditions, can be the switching generator of those biclques, i.e. concepts.

\section{Conclusion}

The recent candidates to be closure operators related to triconcepts are not always consistent with either the definition of closure operator or triconcept (n-concept). We considered partially defined closure operators for triconcept generation that solve the problem. It is easy to obtain their $n$-adic versions and generalise current results. However the open question at the moment is whether recent closure-based algorithms for pattern mining reported in the relevant literature may benefit from this new bit of knowledge. Even though their basic definitions can be refined to fulfill necessary requirements, as we have seen, it might be costly or even intractable. Thus, an interesting prospective result could be a polynomial time check whether the current context is switching generators free (excluding $\emptyset$ ) or has a polynomial number of switching generators; one of the switching generators free examples is $\mathbb{K}=(\{1 \ldots m\}, \ldots,\{1 \ldots m\},=)$. 


\section{Acknowledgments}

The author would like to thank Loïc Cerf, Eirini Spyropoulou, Boris Schminke, Dmitry Gnatyshak, Sergei Kuznetsov, Sergei Obiedkov, Bernhard Ganter, JeanFrancois Boulicaut, Mehdi Kaytoue, Amedeo Napoli, Boris Mirkin, Lhouri Nourine, Engelbert Mephu Nguifo and Jaume Baixeries. The study was implemented in the framework of the Basic Research Program at the National Research University Higher School of Economics in 2015-2017 and in the Laboratory of Intelligent Systems and Structural Analysis. The author was also partially supported by Russian Foundation for Basic Research.

\section{References}

\section{References}

Belohlávek, R., Glodeanu, C., \& Vychodil, V. (2013). Optimal factorization of three-way binary data using triadic concepts. Order, 30, 437-454.

Biedermann, K. (1998). Powerset trilattices. In M.-L. Mugnier, \& M. Chein (Eds.), Conceptual Structures: Theory, Tools and Applications (pp. 209221). Springer Berlin Heidelberg volume 1453 of Lecture Notes in Computer Science. URL: http://dx.doi.org/10.1007/BFb0054916, doi 10 . 1007/BFb0054916

Boley, M., Horvath, T., Poigne, A., \& Wrobel, S. (2010). Listing closed sets of strongly accessible set systems with applications to data mining. Theoretical Computer Science, (pp. 691-700).

Cerf, L., Besson, J., Nguyen, K.-N., \& Boulicaut, J.-F. (2013). Closed and noise-tolerant patterns in n-ary relations. Data Min. Knowl. Discov., 26, $574-619$.

Cerf, L., Besson, J., Robardet, C., \& Boulicaut, J.-F. (2009). Closed patterns meet n-ary relations. ACM Trans. Knowl. Discov. Data, 3, 3:13:36. URL: http://doi.acm.org/10.1145/1497577.1497580, doi:http:// doi.acm.org/10.1145/1497577.1497580. 
Crespelle, C., Latapy, M., \& Phan, T. H. D. (2015). On the termination of some biclique operators on multipartite graphs. Discrete Applied Mathematics, 195, 59 - 73. URL: http://www.sciencedirect. com/science/article/pii/S0166218X15000621. doi:http://dx.doi.org/ 10.1016/j.dam.2015.02.006. 12th Cologne-Twente Workshop on Graphs and Combinatorial Optimization (CTW 2013).

Ganter, B., \& Glodeanu, C. V. (2012). Ordinal factor analysis. In Formal Concept Analysis - 10th International Conference, ICFCA 2012, Leuven, Belgium, May 7-10, 2012. Proceedings (pp. 128-139). URL: http://dx.doi. org/10.1007/978-3-642-29892-9_15. doi:10.1007/978-3-642-29892-9_ 15 .

Ganter, B., \& Kuznetsov, S. O. (2001). Pattern structures and their projections. In Conceptual Structures: Broadening the Base, 9th International Conference on Conceptual Structures, ICCS 2001, Stanford, CA, USA, July 30-August 3, 2001, Proceedings (pp. 129-142). URL: http://dx.doi.org/10.1007/ 3-540-44583-8_10. doi:10.1007/3-540-44583-8\_10.

Ganter, B., \& Wille, R. (1999). Formal Concept Analysis: Mathematical Foundations. (1st ed.). Secaucus, NJ, USA: Springer-Verlag New York, Inc.

Glodeanu, C. V. (2013). Tri-ordinal factor analysis. In Formal Concept Analysis, 11th International Conference, ICFCA 2013, Dresden, Germany, May 2124, 2013. Proceedings (pp. 125-140). URL: http://dx.doi.org/10.1007/ 978-3-642-38317-5_8, doi $10.1007 / 978-3-642-38317-5 \_8$.

Hacene, M. R., Huchard, M., Napoli, A., \& Valtchev, P. (2013). Relational concept analysis: mining concept lattices from multi-relational data. Ann. Math. Artif. Intell., 67, 81-108. URL: http://dx.doi.org/10.1007/ s10472-012-9329-3, doi $10.1007 /$ s10472-012-9329-3.

Ignatov, D. I., Gnatyshak, D. V., Kuznetsov, S. O., \& Mirkin, B. G. (2015). Triadic formal concept analysis and triclustering: searching for optimal pat- 
terns. Machine Learning, 101, 271-302. URL: http://dx.doi.org/10. 1007/s10994-015-5487-y, doi 10.1007/s10994-015-5487-y.

Jäschke, R., Hotho, A., Schmitz, C., Ganter, B., \& Stumme, G. (2006). Trias-an algorithm for mining iceberg tri-lattices. In Proceedings of the Sixth International Conference on Data Mining ICDM '06 (pp. 907-911). Washington, DC, USA: IEEE Computer Society. URL: http://dx.doi.org/10.1109/ ICDM.2006.162, doi:http://dx.doi.org/10.1109/ICDM.2006.162,

Kaytoue, M., Codocedo, V., Buzmakov, A., Baixeries, J., Kuznetsov, S. O., \& Napoli, A. (2015). Pattern structures and concept lattices for data mining and knowledge processing. In Machine Learning and Knowledge Discovery in Databases - European Conference, ECML PKDD 2015, Porto, Portugal, September 7-11, 2015, Proceedings, Part III (pp. 227-231). URL: http://dx.doi.org/10.1007/978-3-319-23461-8_19, doi 10.1007/ 978-3-319-23461-8_19.

Kaytoue, M., Kuznetsov, S. O., Macko, J., \& Napoli, A. (2013). Biclustering meets triadic concept analysis. Annals of Mathematics and Artificial Intelligence, (pp. 1-25). URL: http://liris.cnrs.fr/publis/?id=6292 doi:10.1007/s10472-013-9379-1.

Krolak-Schwerdt, S., Orlik, P., \& Ganter, B. (1994). Tripat: a model for analyzing three-mode binary data. In H.-H. Bock, W. Lenski, \& M. Richter (Eds.), Information Systems and Data Analysis Studies in Classification, Data Analysis, and Knowledge Organization (pp. 298-307). Springer Berlin Heidelberg. URL: http://dx.doi.org/10.1007/978-3-642-46808-7_27 doi: $10.1007 / 978-3-642-46808-7 \_27$.

Kuznetsov, S. O. (2004). Complexity of learning in concept lattices from positive and negative examples. Discrete Applied Mathematics, 142, 111-125.

Lehmann, F., \& Wille, R. (1995). A triadic approach to formal concept analysis. In Proceedings of the Third International Conference on Conceptual 
Structures: Applications, Implementation and Theory (pp. 32-43). London, UK: Springer-Verlag. URL: http://dl . acm.org/citation.cfm?id=645488. 656867.

Lijffijt, J., Spyropoulou, E., Kang, B., \& Bie, T. D. (2016). P-n-rminer: a generic framework for mining interesting structured relational patterns. I. J. Data Science and Analytics, 1, 61-76. URL: http://dx.doi.org/10.1007/ s41060-016-0004-3, doi $10.1007 / \mathrm{s} 41060-016-0004-3$.

Mirkin, B., \& Kramarenko, A. V. (2011). Approximate bicluster and tricluster boxes in the analysis of binary data. In S. O. Kuznetsov, D. Slezak, D. H. Hepting, \& B. Mirkin (Eds.), RSFDGrC (pp. 248-256). Springer volume 6743 of Lecture Notes in Computer Science.

Simovici, D. A., \& Djeraba, C. (2008). Mathematical Tools for Data Mining: Set Theory, Partial Orders, Combinatorics. Springer-Verlag London Limited.

Spyropoulou, E., Bie, T. D., \& Boley, M. (2014). Interesting pattern mining in multi-relational data. Data Min. Knowl. Discov., 28, 808-849. URL: http://dx.doi.org/10.1007/s10618-013-0319-9, doi 10 . 1007/s10618-013-0319-9.

Trabelsi, C., Jelassi, N., \& Ben Yahia, S. (2012). Scalable mining of frequent tri-concepts from folksonomies. In P.-N. Tan, S. Chawla, C. Ho, \& J. Bailey (Eds.), Advances in Knowledge Discovery and Data Mining (pp. 231-242). Springer Berlin Heidelberg volume 7302 of Lecture Notes in Computer Science. URL: http://dx.doi.org/10.1007/978-3-642-30220-6_20, doi 10 . 1007/978-3-642-30220-6_20.

Voutsadakis, G. (2002). Polyadic concept analysis. Order, 19, 295-304.

Wille, R. (1995). The basic theorem of triadic concept analysis. Order, 12, $149-158$.

Zaki, M. J., \& Hsiao, C. (2005). Efficient algorithms for mining closed itemsets and their lattice structure. IEEE Trans. Knowl. Data Eng., 
17, 462-478. URL:http://doi.ieeecomputersociety.org/10.1109/TKDE.

2005.60. doi:10.1109/TKDE.2005.60. 